\title{
Acompañamiento con grupos en Trabajo Social. Un modelo interfocal para la comprensión y sistematización de la praxis
}

\author{
Support with Groups in Social Work: An Interfocal Model \\ for the Understanding and Systematization on Praxis \\ Belén ARIJA GiSBERT \\ Universidad Complutense de Madrid \\ belenarija@hotmail.com
}

Sólo se ve bien con el corazón.
Lo esencial es invisible a los ojos
Antoine de Saint Exupéry

Recibido: $14 / 03 / 2012$

Revisado: 27/03/2012

Aceptado: 28/05/2012

Disponible on line: 03/08/2012

\begin{abstract}
Resumen
El trabajo pretende reflejar la mirada de la autora sobre la relación de ayuda, y en particular sobre el acompañamiento con grupos en Trabajo Social. Asimismo, aporta algunas experiencias actuales y rigurosas, para que sirvan de estímulo a nuevas iniciativas de participación grupal y comunitaria. Es una invitación a imaginar y a levantar todos juntos nuevos escenarios de pertenencia constructiva.

Además, en coherencia con la mirada o el marco teórico que denomino interfocal, la autora comparte una propuesta de crónica grupal o un modelo de sistematización de la praxis. Una alternativa reflexiva que se puede adaptar y matizar en los diferentes contextos donde se utilice, como ya se está haciendo, con la intención de ayudar al grupo a comprender y acceder al transfondo. Como profesionales se trata de pensar juntos, cooperar y sentir el grupo de modo reflexivo con el propio grupo. Defiende que la metodología con la que trabaja ha de basarse en la investigación-acción participante. Desea que la teoría se viva más como un apoyo y una guía y no como un universo desconocido y alejado de la práctica. Se trata de tender puentes entre el mundo académico y el ámbito profesional; de dibujar, entre todos, un horizonte nuevo y esperanzador, para los profesionales y para las personas con las que trabajamos, en el que el Trabajo Social con grupos tenga una presencia central y necesaria en estos momentos de crisis y de cambio.
\end{abstract}

Palabras clave: Trabajo Social con grupos, pertenencia, encuentro, teoría, acompañamiento, afinidad, reflexión, sistematización, vínculo, comprensión, participación.

\begin{abstract}
This article presents the point of view of the author on the help relationship, particularly on supporting groups in Social Works, It provides some current and rigorous experiences as well, that serve to encourage new initiatives for groups and community participation. It is an invitation to imagine and stage new scenes of constructive ownership. Further, congruent with the so-called intermodal perspective or theoretical framework, the author shares a proposal of group chronicling or a praxis systematization model. An alternative for reflection that can be adapted and tailored to different contexts where it is already being used with the intent to help the group understand and access background. As professional, we seek to thing togheter, cooperate and feel the group reflexively with the group itself. She defends the idea that the methodology being used must be based on participant action-research. She shopes that the theory will live as as a support and a guide and not as an unknown universe far from practice. The author seeks to build bridges between the academid and professionaol worlds, to draw, among other things, a new, hopeful perspective for professional and for the people with whom they work, in that Social Work with grups will have a central, necessary presence in these times of crisis and change.
\end{abstract}

Key words: social works with groups, ownership, encounter, theory, support, affinity, reflection, systemization, link, understanding, participation.

Referencia normalizada: Arija Gisbert, B. (2012): «Acompañamiento con grupos en Trabajo Social. Un modelo interfocal para la comprensión y sistematización de la praxis». Cuadernos de Trabajo Social, 25(2): 427-438. 
Sumario: 1. Pertenencia constructiva por afinidad. 2. Ejemplos de buena praxis en Trabajo Social con grupos. 3. La coordinación en el acompañamiento con grupos. Algunos apuntes para iniciar. 4. El marco teórico: una mirada interfocal. 5. El esquema para la sistematización de la praxis interfocal. 6. Conclusiones: ventanas reflexivas y puentes. 7. Referencias bibliográficas.

\section{Pertenencia constructiva por afinidad}

Lo primero es el vínculo, sin el contacto y la cercanía la vida se apaga. Muchas personas se mueren solas en las casas o en la calle, invadidas por la pena. Os invito a leer los experimentos de Spitz (1999) cuando planteaba que los niños sin contacto se consumen, sin apego amoroso se mueren los pequeños en los orfanatos. Permitidme este comienzo tan directo, es tan abrupto como la vida en ocasiones.

El grupo puede regalar vínculos y aceptación, proporcionar ideas para abordar problemas materiales, así como identidad, soluciones de reciprocidad, compañía, aprendizaje, desahogo, pertenencia, sano poder, reconocimiento y cariño. Esa necesidad vital que tan pocas veces se legitima y se nombra.

Nos manifestamos en el afecto y en el poder bien entendido, necesitamos empoderarnos de valor y de valía. Ni los profesionales ni los ciudadanos queremos vivir en la impotencia del vacío infértil del que habla la Gestalt (Peñarrubia, 1998, p. 109). Es saludable la creación colectiva, así como descansar en la presencia de otra mirada y afirmarse en el eje diferenciado de uno mismo, porque aislarse del «otro amenazante» de Sartre es la locura.

El grupo es su inmenso potencial, su sano poder para que las personas rescaten la viveza que proporciona lo colectivo, la sonrisa y el gesto, la ilusión y la esperanza. Apegarse a la vida, enraizando con otros en la ayuda mutua, es hacer prevención auténtica. La dimensión social nos compete a los trabajadores sociales por identidad y por ética. En los barrios, en las calles, con la comunidad, con los individuos y las familias, en la relación de ayuda dentro de las organizaciones de Servicios Sociales, en los equipos profesionales. Lo grupal, concebido como un imaginario de armonía o de conflicto, como un espacio sano que habitamos, como un espacio tóxico o como un escenario que nos es indiferente. Las polaridades navegan siempre entre matices.
El grupo es germen y cosecha para la acción social: trabajar juntos para lograr cambios más allá de uno mismo, grupos de acción y reflexión surgidos a partir del 15M, grupos en áreas tales como vivienda, educación, salud, consumo responsable y desarrollo comunitario. Todo hace grupo. El grupo es y nos necesita: ya existe en los centros residenciales, donde viven las personas, ya sean niños, mayores o con problemáticas; también en las salas de tratamiento de enfermos crónicos o en los hospitales; en la calle, como chavales alrededor de las drogas; entre los sinhogar en la soledad sonora del bu1licio, etc. Nos compete a los trabajadores sociales escuchar y acoger propuestas que contribuyan a que las personas amplíen su red social y se apoyen recíprocamente.

Miguel Marinas (2001) anticipa alternativas al consumo, nos habla de «afinidad», así como del consumo con su despilfarro, simulacro, mentira; de satisfacción inmediata, del bótox, la moda, etc. Con la sociedad de consumo hemos cambiado el verbo compartir por el verbo tener.

En tiempo de soledad, vacuidad y precariedad, démosle su valor al grupo. La pertenencia debe procurar ser un espacio constructivo partiendo de lo común. No sirve la participación impuesta o la exigencia, sino la motivación de estar en lo afín, a partir de la no obligatoriedad. Estimular la creación de alternativas para resolver lo relacional, lo material y lo que dé sentido a la vida; así como potenciar las salas abiertas para el encuentro y la creación (Arija y Herrero, 1998, pp. 181-201). Volver a las «casas del pueblo», a las reuniones fructíferas de intercambio entre organizaciones es reunirse para reflexionar y sentir juntos, con las personas, en un intento de cooperar para que florezcan las iniciativas participativas. Lo individual, lo grupal y lo comunitario no son niveles de intervención disociados, sino dimensiones entrelazadas que deben nutrirse recíprocamente en un intento de acompañar a las personas. 
Hemos pasado a formar parte de un sistema que deshumaniza la relación de ayuda y marca insanas relaciones de poder. García (2012) señala, al respecto, que:

La dimensión colectiva y cooperativa de la educación y la intervención social es un aspecto que rara vez se cuida. La forma en que las instituciones públicas interaccionan con los ciudadanos, que depende en último término de los profesionales que trabajan en sus puertas de entrada, tiende a ser, a mi parecer, vertical e individualizada (p. 217).

Las vanguardias de la profesión, como las del arte, son contrapuntos a la verticalidad y plantean una identidad más democrática, horizontal, una ética más comprometida. Trabajadores sociales que intervienen en red, con la participación de las personas, desarrollan propuestas valiosas en las que se entreteje el acompañamiento grupal, comunitario e individual. Profesionales que se forman, se supervisan y viven la teoría como un mapa que alumbra el camino y que reconstruyen gracias a la experiencia. Lo que nos compete es estar; la presencia la requiere el acompañamiento, con cercanía, respeto y conocimiento. El grupo nos afecta en cualquier ámbito y con cualquier colectivo; y es un deber moral impulsar lo nuevo y reconducir lo que va a la deriva y necesita ser escuchado. Es nuestra responsabilidad recrear nuestra identidad profesional, pero los contextos grupales no son de los educadores, de los monitores o de los terapeutas ocupacionales. ¿Para qué el corporativismo a costa de la identidad?

Como profesora de Trabajo Social con grupos y supervisora de prácticas en una universidad pública, tengo la responsabilidad de compartir lo creado en un proceso de acción-reflexión con la participación de muchas personas: grupos de formación, grupos en el acompañamiento, grupos de supervisión, etc.

Debemos invitar a todos a salir de los despachos y a tomar la calle, tomarla en el sentido de participar con las personas en el espacio que ya nos pertenece. El reino no puede ser de presuntos duques o prevaricadores que se montan palacios y feudos, usurpando lo que es propiedad de la ciudadanía. Teresa Zamanillo (2011) afirma que debemos basar nuestra intervención en «unos criterios éticos (...) cuya esencia se encuentra en la responsabilidad individual (...) Es el cuidado de uno mismo, en primer lugar, como condición sine qua non, lo que nos facilitara el cuidado de otros.» (p. 9). «El cuidado de uno, de los otros y de la institución» es la propuesta que esta autora plantea siguiendo a Ricoeur (p.14) y cuya idea comparto. Se ha de adoptar una metodología que implique a los protagonistas en el acto de conocer y de crear su propia travesía grupal y aplicar la investigación-acción-participante, el método creado por Kurt Lewin.

Para generar un grupo es preferible colocarse en círculo que hacerlo en fila. Contamos con el seminario y el aula magistral, la sala y el cuarto de estar, la calle, el parque, el campo y las plazas, además de que disponemos del despacho. Se trata de ampliar el universo de posibilidades, de integrar contextos y dimensiones y de admitir sugerencias y críticas, siempre que vayan acompañadas de alternativas. Respetemos las preferencias, los posicionamientos diferentes, las capacidades diversas y creativas de los profesionales porque juntos nos abrimos a la tormenta de ideas. Ha llegado el tiempo de pensar, sentir, dialogar y crear de manera cooperativa. Podemos diferenciarnos los unos de otros con respeto. He escuchado en muchas ocasiones a José Luis Valero, terapeuta gestalt y coordinador de grupos socioterapéuticos, que dice: «El unívoco discurso de la excelencia oscurece la dignidad de la suficiencia, la dignidad de ser suficientemente buenos profesionales».

Es momento de trazar el círculo y escucharnos, a pesar del miedo a lo desconocido. El miedo es una emoción legítima; es mejor acercarse con temor a proponer un grupo que quedarse paralizado por miedo tras la mesa de un despacho lleno de papeles, como único medio de ejercer el Trabajo Social. Animo a los más valientes - me refiero a los temerosos - ya que sólo es valiente quien siente miedo y con miedo podemos, desde lo «suficientemente bueno», proponer experiencias grupales y juntarnos para aprender a resolver problemas y dilemas éticos. Unirnos también para hallar sentido a la vida, que no es poco. Aurora Castillo, amiga y profesora de Trabajo Social, cuando recuerda a Victor Frankl, comenta que las personas necesitan hallar un significado a sus vidas y los trabajadores sociales debemos movilizar la ilusión, el sentido y, si se puede, la alegría. Es importante ser técnicos y humanos, cando 
nos disponemos a acompañar los procesos de mejora. La reflexión es siempre un proceso abierto, un diálogo creativo en un determinado momento, es bueno el fluir del intercambio de experiencias y ayuda usar una didáctica del ejemplo.

\section{Ejemplos de buena praxis en Trabajo So- cial con grupos}

Rosalía Martínez, trabajadora social, afirma:

La gestión de prestaciones y servicios es necesaria en Trabajo Social, al igual que gestionar los tiempos y los escenarios, las estrategias, las capacidades y las actitudes, los conflictos y las nuevas propuestas, etc. La gestión forma parte de nuestro trabajo.

Rosalía tiene formación en mediación sistémica y en grupos, lleva años trabajando en distintas organizaciones; como antropóloga utiliza técnicas cualitativas y de análisis de los procesos grupales; trabaja en una residencia de protección de menores y pretende que la sala de encuentro sea una verdadera escuela de padres, que las visitas de los padres y madres a sus hijos sean de calidad; además confía en el grupo $\mathrm{y}$ en la presencia de profesionales. Laura $\mathrm{Ca}-$ lles fue supervisada por Rosalía y se está formando en terapia gestalt y sistémica, porque pretende desarrollar una escuela de padres en el colegio de primaria donde es trabajadora social. También Javier Baeza y su equipo de la parroquia de San Carlos Borromeo de Vallecas son un ejemplo de buena praxis. Hay trabajadores sociales que están realizando, voluntariamente, acompañamiento psicosocial y socioeducativo con rigor teórico y metodológico, basado también en la confianza y es admirable contemplar la confianza que despiertan su presencia en las personas que malviven en El Gallinero, en La Cañada Real: sin agua, sin luz, en un contexto vital inundado por la miseria. Confianza que se han ganado a costa de estar presentes con respeto y acercarse a las familias y a los grupos con educación cívica.

Todos aquellos profesionales de la relación de ayuda que entran en los contextos invasivamente con paternalismo y control y con la bandera de la amenaza simbólica del poder, sea en el contexto que sea, hallarán a personas asustadas que se esconden y obedecen con miedo, o reaccionan retirándose o manifestando hostilidad. Esos «técnicos» se creen que «los pobres» son marionetas para modelar, sin pararse ni siquiera en intentar comprender sus esquemas culturales ni mucho menos tratan de empatizar con sus precarias condiciones vitales.

Para cimentar lo nuevo, sin duda, hay que retirar los escombros y ser constructivos. La pertenencia constructiva por afinidad con la cuestión del género, la cooperación y el arte en sentido amplio y como aspectos transversales ha de basarse en una «ética del cuidado con límites», tal y como lo plantea Silvia Navarro (2004, p. 209). Tamara de la Mora tiene formación sistémica y en Trabajo Social con grupos, ha desarrollado, entre otros, en la Asociación Tyerra y el Ayuntamiento de Galapagar el proyecto $E n$ la conciliación nuestros mayores cuentan. Uno de los objetivos de este grupo es reconocer el papel importante que desempeñan los abuelos $\mathrm{y}$ abuelas en la conciliación de la vida laboral y familiar de sus hijos/as cuando se encargan del cuidado de sus nietos.

Marta García hace posible el encuentro y el apoyo mutuo en una asociación de Vallecas, acompañando a un grupo de mujeres gitanas, $y$ es a través del cante flamenco como se encuentran. «La alfabetización entra mejor a las mujeres si la acompañamos de bulerías». A la vez resuelven juntas aspectos que les crean conflicto.

Cristina Alonso lleva a cabo, en un centro de Salud Mental, una alternativa de recuperación psicosocial con personas con enfermedad mental grave: un grupo de participación voluntaria, que utiliza como instrumento la escritura creativa. Consiguen juntos crear vínculos y relaciones de confianza que les permiten salir del aislamiento, tener voz y superar las dificultades del estigma que supone la enfermedad mental. Formada en el modelo sistémico, trabaja bajo el enfoque de Arte y Trabajo Social.

Las profesoras del Departamento de Trabajo Social y Servicios Sociales de la Complutense, $\mathrm{M}^{\mathrm{a}}$ Victoria de la Heras, Maribel Nebreda, y Maribel Martín, crearon, con su apoyo, una iniciativa participativa - Socialuloidecon objeto de generar un espacio de aprendizaje complementario con la actividad puramente académica. A través del cine forum se posibilitó el debate sobre la realidad en la que actúa el Trabajo Social. Un encuentro entre cineastas, 
expertos, profesionales, profesores y estudiantes, Socialuloide constituyó un puente, entre lo académico, el Arte y el Trabajo Social profesional.

Teresa García Sánchez trabaja en una parroquia de Tetuán $\mathrm{y}$, partiendo de un modelo rogeriano, supervisa su trabajo y se esfuerza por cuidarse para cuidar; confía y potencia el trabajo en red con otras instituciones del distrito. Lleva un grupo de danzas del mundo y, a partir del encuentro en el grupo, las mujeres cuidan su salud, se divierten y amplían su red de apoyo. Coordina grupos de consumo responsable con las familias, para encontrar vías que les permiten encarar lo económico y lo ecológico. La institución en la que trabaja apoya su iniciativa y las personas con libertad participan en los grupos que ella propone, son circuitos sanos que se retroalimentan. Julia Tabernero conduce grupos socioeducativos de ocio y tiempo libre con menores con riesgo de exclusión, para que aprendan a relacionarse de forma cooperativa. Le sirven la filosofía de Freire y la Gestalt, Foucault y Bourdieu, para abordar temas de poder y violencia. Beatriz Luis, desde el ámbito penitenciario, ha impulsado grupos de comunicación en sala abierta, de danza terapéutica y ocio creativo, tiene formación teórica y experiencia en grupo gestáltico y en psicodrama. Supervisa su trabajo y se esfuerza por crecer como persona.

Los bancos de tiempo proliferan como grupos basados en la reciprocidad; además de intercambiar tiempo, se hacen amigos y se puede realizar desarrollo comunitario. A mi modo de ver, debe coordinarlos un profesional con talante mediador, que ayude a su gestión y a equilibrar los trueques. Otros ejemplos proceden de otras iniciativas de grupos que ponen en relación Arte y Trabajo Social; de la prevención de drogas a través del deporte; del apoyo comunitario y vecinal; de experiencias de ayuda mutua entre personas con la misma problemática; de grupos de duelo para trabajar las pérdidas, muertes y separaciones o los grupos de autogestión, con huertos urbanos. etc.

\section{La coordinación en el acompañamiento con grupos. Algunos apuntes para iniciar}

Prefiero hablar de acompañamiento, porque acompañar es ir junto a las personas, al lado del grupo, procurar la interdependencia de sus miembros; seguimiento me remite a ir detrás. Las palabras conforman cosmovisiones: ser compañeros de viaje implica una relación cercana y sólida; una relación diferenciada por lo específico que tiene la función de coordinar, cuya responsabilidad es saber guiar el barco, estar presente y pendiente de los elementos, como los mapas que nos ayudan a no perder el rumbo y navegar al ritmo del proceso grupal; juntarse para un fin marcado por el grupo, con unas normas flexibles y sólidas, y con la cooperación y reflexión de todos. Adentrarse en la travesía con sus momentos de armonía y conflicto, por un camino basado en la corresponsabilidad y en la acción reflexiva.

Lo primero es crear vínculo, propiciar un clima de confianza cada vez que nos sentemos en el círculo del grupo. Sin confianza es muy difícil entregarse a un proceso, sea el que sea, hay que empezar escuchando, partir de lo que quieren contarse unas personas a las otras y a la coordinadora; a este momento hay que dedicarle su tiempo y su espacio y a escuchar con el marco de análisis de lo intrapersonal, lo interactivo y lo grupal (López Yarto, 1997) y lo sociocultural, con la apertura que señala la interfocalidad cuando contempla el arte y la actitud.

Referentes como Bolwvy (1989), Howe (1997) o Rogers $(1961,1973)$ dan buena cuenta de lo importante que es el vínculo, como base segura, la empatía y la aceptación; las personas lo necesitan para tomar fuerzas y confianza, para impulsar su vida y se trata de partir de la generación de un sano vínculo con el grupo que nace o que lleva ya un recorrido.

Se trata de ver juntos para qué estamos reunidos. La primera sesión marca las normas de relación y ayuda ya a vislumbrar objetivos grupales. Con mi función diferenciada puedo acompañar, ser acompañante, Me gusta el concepto, lo aprendí de Rogers (1961), procede del latín, panis, acción de comer de un mismo pan (Corominas, 2006, p. 162).

Ser los trabajadores sociales compañeros de viaje es adentrarse en unas coordenadas que requieren pararse a mirar la vida de las personas, no sólo importan los problemas, sino sus capacidades y actitudes facilitadoras. Trabajar por lo saludable, sin negar las dificultades, ha de ser otro foco al que atender, se trata de una cuestión apasionante, y como toda pasión, necesita prudencia y racionalidad así como mapas teóricos, 
con creatividad, brújula y focos interconectados. En ocasiones como profesionales nos equivocamos - ¿quién no? - pero el error, cuando lo vemos, se convierte en una oportunidad para cambiarlo. Como coordinadora formo parte del grupo y debo auto-observarme; formo parte del campo de fuerzas al que se refiere Lewin (1988); me doy cuenta y me responsabilizo: es un imperativo gestáltico; se trata de escuchar con la atención flotante, la contratransferencia, que impone el psicoanálisis (Laplanche y Pontalis, 1994; Vals, 1995). Me superviso, en primera persona, y como tal debo atender y cuidar lo que se me mueve en el acto de coordinar un grupo, de ahí que la supervisión sea una forma imprescindible de cuidarnos y cuidar nuestro trabajo. Cada cual ha de ver dónde tiene que pulir sus actitudes, su propia persona y cómo tiene que seguir completando su formación. La teoría, los autores, los modelos, son focos de luz que alumbran el contexto. Cuanta más formación tengamos más conocimiento, estrategias, técnicas, sana actitud y arte pondremos en la conducción de grupos. Carezco de un recetario de actuaciones, no sirve el manual porque cada grupo es un mundo y prefiero la reflexión colectiva para aprender significativamente y ver juntos cómo proceder.

Como coordinadora es preciso sentarse a pensar a ratos en soledad, con el lápiz y el cuaderno de campo. La riqueza de pensar colectivamente con el grupo sobre la marcha de su proceso, reflexionar con el co-coordinador y la observadora y la supervisora, ofrecen un punto de vista diferenciado. Durante seis años fui observadora de algunos grupos operativos que Teresa Zamanillo coordinaba en la asignatura de Trabajo Social con grupos, Aprendí vivencialmente a estar en este rol, fue un gran aprendizaje, entre otras cuestiones, del silencio, el potencial de la intersubjetividad y la transparencia.

La riqueza de la experiencia está en la multitud de ojos que miramos lo que pasa. En la mirada de Teresa que es la que lleva la coordinación y el peso de la experiencia, en la mía como observadora y en la de los alumnos observadores que aportan con sus descripciones y reflexiones una gran riqueza a la dinámica $\mathrm{y}$, cómo no, en la de los propios participantes del grupo operativo (Arija, 2000, p. 131).

No se aprende a conducir sólo con saberse el manual, el proceso debe ser simultáneo de la ex- periencia a la teoría, querer esperar a saber teoría y después formar grupos, es posponer, aliarse con la pereza, las excusas o los miedos $\dot{\zeta} \mathrm{Y}$ si hacemos grupo y a la vez nos formamos y nos supervisamos? Comencemos la travesía del grupo con atrevimiento y temor, con mapas y con apoyos.

\section{Marco teórico. Una mirada interfocal}

Para estudiar y comprender separamos los elementos analíticos, sabiendo que en las escenas de la vida no es posible hacer esta división que hacemos al investigar o al sistematizar la praxis, ya que hay infinidad de variables que se interrelacionan. La práctica es más amplia y rica de lo que puede ofrecer una descripción e interpretación, por muy rigurosa que sea. El mapa no es el territorio, el mapa orienta la enormidad del territorio. Popper (1982) nos señala que la teoría es como una red que lanzamos al agua (la realidad), el tamaño de los agujeros de esta red permitirá atrapar ciertos peces, no otros, lo que no significa que lo que queda fuera de la red no exista. Con esta metáfora queda claro que recortamos la realidad y que la observación, descripción e interpretación son actos limitados.

Confío en una mirada, que denomino interfocal, que contempla diversas teorías aparentemente opuestas y que invito a poner en relación $\mathrm{y}$ en hermandad. Es viable integrar modelos teóricos que parecen antagónicos, esta integración implica optar por una elección teórica. Mi opción, como antropóloga social, es lo cualitativo como vía de investigación y el estructuralismo de Strauss hermanado con el funcionalismo de Malinovski. Margareth Mead con Ortega y Gasset. Ellos y otros conviven en mi marco, lo necesario es enfrentarnos a preguntas mucho mejor que enfrentar modelos teóricos y generar guerras en el ámbito del saber. Hacer investigación rica en variables, siguiendo a Popper y la falsación de hipótesis, a Bourdieu y sus rupturas epistemológicas, y a Jesús Ibáñez para comprender el análisis de los discursos y el grupo de discusión entre otros referentes y entrenarmos tenazmente en la observación participante y en el estudio.

Confío en la actitud y visión antropológica de las teorías humanistas que consideran que el hombre es un ser en transformación. La Gestalt y el psicodrama psicoanalítico, su presencia y su técnica, ayudan a mirar al individuo en 
el grupo y a trabajar formas de estar. El existencialismo y su idea de libertad puede hermanarse con el psicoanálisis y su enfoque determinista de lo intrapsíquico que, a su vez, debe interactuar con una mirada sistémica que se fija en las relaciones.

La persona es el vínculo y a la vez es lo indivisible. La pregunta de si el individuo existe sin relación y sin contexto es algo que aprehendí conversando con Norberto Barbagelata, psiquiatra y terapeuta sistémico, para quien la clave está en preguntar al psicoanálisis por su carencia en mirar lo relacional y contextual, y a la teoría sistémica cuestionarle su limitación en mirar lo intrapsíquico del individuo. Barbagelata une a Minuchin y a Freud, a Bateson y a Lacan en un diálogo entre sus modelos.

Desde la perspectiva interfocal debemos preguntamos por las sombras en nuestra mirada teórica y en nuestras capacidades; así como por nuestros temores y nuestra actitud; por lo que de obstaculizador tiene nuestro carácter; y por las creencias incuestionadas. También debemos reconocer nuestros talentos, nos interpelan la ética y los valores. El arte puede ser un foco que alumbra nuestro camino. La interfocalidad contempla la unión de lo psicosocial con lo ético y filosófico, que entronca a su vez con lo político, que es a la vez ético y estético; $\mathrm{y}$ este último aspecto conecta con el cuerpo, que es el sostén de la vida. La interfocalidad no se emparenta con el eclecticismo ni con el «todo vale», rebasa la mirada del enfoque ecológico o el constructivismo, no es sólo la interrelación o la construcción de multiversos o la interdisciplinariedad: es la integración y la hermandad de modelos y elementos de análisis, disciplinas, creatividad, artes diversas y actitudes, para navegar en el complejísimo mundo del Trabajo Social con grupos. Es un marco atravesado por la emoción, el pensamiento, la actitud y el arte. Ética y estética, el zen y la danza, el cuerpo y las artes, la medicina y la ecología, además de las habilidades sociales. Estos focos constituyen alternativas complementarias a lo clásico y ortodoxo.

Necesitamos más coexistencia y menos luchas de poder. Menos axiomas y verdades finales y que cada cual se pregunte qué aporta al conflicto, si es que forma parte de algún conflicto grupal. Es una vía auto-responsabilizada. Invito a que todos lo hagamos, porque es sano hacerse cargo de los errores, pedir disculpas al grupo o a alguna persona, si se ha obrado mal. Mi propuesta es: "Miremos la viga en el ojo propio y será mejor para todos», porque no sólo es suficiente la negociación como forma de resolución de conflictos, hay que hacerse cargo de la responsabilidad de cada uno de nosotros, pero no sirve atraparse en culpabilidades paralizantes o señalamientos acusatorios hacia fuera, que son caras de la misma moneda. Ni verdugos ni víctimas, ni salvadores ni salvados: seamos profesionales responsables, que asumimos nuestra parte del problema y cooperamos para su resolución. Es una de las condiciones indispensables para la interfocalidad.

Dejemos las idealizaciones y las exigencias, las armas y las trincheras, busquemos la autoresponsabilidad, la cooperación y el respeto a los distintos enfoques. Lo peor de todo son las personas-profesionales que llevan la orquesta del subgrupo determinado, que en un lugar con poder abusan del mismo, ostentan rigurosidad y carecen de humanidad, instrumentalizan a las personas y les exigen sin escrúpulos.

A continuación, expongo los elementos de análisis hacia los que pretendo orientar la mirada: por un lado, son los objetivos y normas del grupo como elementos estructurales a atender; y por el otro, se constituyen en referente con su esquema de los cuatro grandes tipos de fuerzas que intervienen en los grupos (López Yarto, 1997), esquema valioso para unir enfoques y categorías analíticas. Estas cuatro fuerzas son:

1. Las fuerzas intrapersonales: la mirada al individuo y los modelos humanistas de Rogers, Gestalt y el enfoque de las necesidades de Schutz. El modelo psicoanálitico de Freud y otros desarrollos del psicoanálisis, así como los mecanismos de defensa: proyección, identificación, racionalización y negación.

2. Las fuerzas interpersonales o la mirada hacia la interacción. La pertenencia, el control y el afecto en clave relacional, se apoyan en las teorías: Teoría de la comunicación del modelo sistémico, Teoría del campo de Lewin: y la de los roles con el enfoque del liderazgo funcional; de las actitudes y los roles en el psicodrama. El clima grupal basado en la tensión o distensión del ambiente. El conflicto, la toma de decisiones, la negociación y la mirada autoresponsable en la resolución de conflictos. 
3. Las fuerzas grupales generadas por el pasado e historia familiar: la relación con la autoridad y el eje vertical común a todos. Las teorías de las que deriva el modelo psicoanalítico grupal: la mirada de Bion y los supuestos básicos. Anzieu y la Escuela francesa de psicodrama psicoanalítico que contempla un eje regresivo, la transferencia, y un eje actual, el tele moreniano. El enfoque psicosocial de Pichon-Rivière y sus elementos analíticos del cono invertido. Las ansiedades básicas: miedo al ataque y a la pérdida.

4. Las fuerzas que López Yarto denomina «los valores». Considero que además de los valores y lo cultural se incluyen en este apartado también las posturas políticas y éticas, así como los marcos sociológicos, entre otros.

A la hora de estudiar un grupo realizamos observaciones e hipótesis que se registran en un diario de campo. Se trata de aprender a identificar aspectos que nos van a posibilitar llegar a un análisis diagnóstico del proceso grupal. Podremos entonces decidir sobre qué elementos incidir para ayudar al grupo a caminar hacia su objetivo.

El esquema para la sistematización que presento a continuación, plantea preguntas basadas en diferentes elementos que en muchos casos van a ser definidos y formulados en términos de polaridad. Es propicio tender puentes entre las teorías de la intervención y los profesionales que están en la base. Espero que cada cual, después, vaya a las fuentes a profundizar sobre lo que, como en un esbozo, expongo en este escrito. Este modelo de sistematización de la praxis grupal, con las adaptaciones necesarias a cada ámbito, lo utilizan distintos profesionales y en distintos contextos. Por ejemplo, para analizar equipos profesionales en proceso de supervisión. En la actualidad, también se usa en la guía para la supervisión educativa del practicum de Grado en la Escuela Universitaria de Trabajo Social de la Universidad Complutense de Madrid, como parte de la actividad para el trabajo en equipo; así como en mi grupo de alumnos de la asignatura de Fundamentos del Trabajo Social con grupos, en grupos de formación de profesionales, en el acompañamiento psicosocial y socioeducativo y en grupos de acción social.

Lo difícil no es imaginar una actividad grupal y plantearla. Cambiar la mentalidad y, con ello, aspectos de la identidad profesional, es el camino.

\section{Esquema para la sistematización de la pra- xis interfocal}

Nombre del grupo: (Tipo de grupo, a quien se dirige y su objetivo General):

\section{Organización:}

Aspectos contextuales:

Fecha de reunión:

Duración:

Presentes:

Ausentes:

Coordinador/s:

Observador/s:

Objetivo de esta sesión:

Grupograma. Es el nombre con el que denomino al dibujo del grupo en su momento de proceso. La representación de los subgrupos desde un modelo sistémico, el haz de relaciones de ese momento grupal según la teoría de la comunicación, las actitudes de los miembros del grupo basado en modelos humanistas y psicoanalíticos, El supuesto básico de Bion (1980) simbolizado, lo intrapersonal, los roles funcionales, los modos de estar de todos los implicados en el tejer del grupo. Se trata de dibujar los elementos plasmando su simbología: la pertenencia, poder y afecto, además de las redes afectivas que la sociometría nos aporta, los mecanismos de defensa. etc. El grupograma como dibujo grupal, será el instrumento del que se vale la interfocalidad como una herramienta comprensiva.

1. Mirada hacia el objetivo grupal ¿Cómo cada miembro contribuye al cumplimiento del objetivo o a «la formulación anticipada de resultados que se quieren producir? (Zamanillo, 2008, p. 202) ¿De qué manera se desarrolla la cooperación o contribución individual a la tarea del grupo? (PichonRivière, 1985). 
2. ¿Qué normas o reglas de comportamiento observamos? (Zamanillo, 2008, pp. 183-200) ¿Qué normas son manifiestas y explícitas, claras y expresadas? ¿Cuáles son aquellas normas implícitas, que no se dicen, y se detectan a partir de lo que se percibe como adecuado o inadecuado? ¿Cómo es la ética que encierran las normas? ¿Qué podemos decir de la responsabilidad de cada individuo?

3. En cuanto a la pertenencia (Schutz, 2001) ¿Cómo es la inclusión y exclusión de los miembros del grupo? ¿Hay personas que están fuera? ¿a quién se excluye e incluye? ¿Cómo se incluye o excluye cada uno del grupo? ¿Cómo se está dentro o fuera? (López Yarto, 1997. Pichon-Rivière, 1985).

4. Desde la teoría de la comunicación del modelo sistémico (Watzlavick, 1985). ¿Qué mensajes se refieren a la relación entre los miembros? ¿Qué aspectos no verbales nos otorgan información de la relación? ¿Cómo se establecen relaciones simétricas (donde prima la igualdad) y cómo se dan interacciones complementarias (donde prima la diferencia)? ¿Observas la existencia de subgrupos? ¿Observamos alianzas? (unión para la consecución de un fin). ¿Entre quiénes y cómo se producen? ¿Observamos coaliciones? (unión para la consecución de un fin y en contra de otros) ¿Entre quiénes y cómo se establecen estas interacciones y comunicaciones?

5. En cuanto a la polaridad dependencia-independencia de los miembros del grupo ¿Cómo es la integración y el grado de tolerancia de las diferencias? ¿Cómo es la diferenciación entre lo racional y lo emocional? (Bowen, 1991).

6. En cuanto a la variable afecto, la sociometría y el análisis del tele (atracción- rechazo) (Moreno, 1966; Pichon-Rivière, 1985) ¿Qué sentimientos y actitudes observamos que nos den cuenta de la atracción y rechazo y de la cercanía o lejanía de cada miembro del grupo con el resto?
7. En relación con el conflicto que va desde la diferencia de pareceres hasta luchas y tensiones afectivas. (Zamanillo, 2008, pp. 227-243) ¿Hemos observado conflictos basados en rivalidades o luchas interpersonales? ¿Cómo se manifiestan? ¿Se resuelven los conflictos? ¿Cómo es la gestión y manejo del conflicto? ¿Se obvian y se tapan? ¿Se responsabiliza cada uno de su parte?

8. ¿Cómo es el modo de toma de decisiones dentro del grupo? ¿Quién o quiénes toman decisiones? ¿Se negocia? (Napier, 2000).

9. El poder desde Foucault ¿Se ven juegos de fuerzas en relación con el poder? Schutz (2001) habla de control. ¿Se dan juegos de dominio/sumisión?

10. Clima ¿Observamos tensión o distensión?

11. Mirando las relaciones con la autoridad o el eje vertical (Bion 1985; Valiente, 1987) Psicoanalisis grupal. ¿En cuál de los tres supuestos básicos inconscientes está el grupo en este momento?:

- Dependencia: ¿Dependen todos de un líder del que esperan protección y todas las soluciones?

- Ataque y fuga: ¿Se atacan, pelean, huyen de la tarea buscando enemigos y chivos expiatorios? ¿El líder es combativo?

- Emparejamiento: ¿Se juntan en parejas esperando soluciones futuras?

12. En cuanto a los roles desde una perspectiva funcional, Bales (1970) y Benne y Sheats (1948) entienden que liderazgo es todo acto que ayude al grupo a realizar sus tareas o a mantenerse unido afectivamente. ¿Hay personas que centran la tarea? ¿Hay personas que velan por la unión afectiva del grupo, por la cohesión? ¿Observamos actitudes obstaculizadoras, que sólo miran por intereses propios? 
13. Lo intrapersonal y humanístico. El individuo en el grupo. Las miradas gestáltica y rogeriana (Moreno, 1966; Anzieu, 1971). Los roles psicodramáticos y psicodrama psicoanalítico. La heteronimia. Las actitudes y los modos de estar de cada uno. La autoobservación de cada miembro del grupo. ¿Qué actitudes facilitadoras, obstaculizadoras, capacidades, necesidades y temores observo en cada miembro? (apoyo, temor, falsedad, cooperación, irresponsabilidad, implicación, rivalidad, mediación, contención, etc.).

14. ¿Observamos mecanismos de defensa inconscientes? (Vals, 1995; Laplanche, 1994):

- Proyección: «Te pongo lo mío».

- Identificación: «yo como tu».

— Negación: «eso no pasa».

15. Aprendizaje ¿En qué hemos avanzado como grupo? ¿Qué fortalezas observamos que tenemos? ¿Qué hemos aprendido? ¿Qué interrogantes nos planteamos? ¿Hemos detectado ansiedades básicas? ¿Miedo al ataque, a ser juzgados o atacados o Miedo a la pérdida del esquema anterior? (Pichon- Rivière, 1985; Kisnerman, 1997).

16. Como coordinador/a ¿Cómo se observa y auto-observa la inclusión en el grupo? ¿Cómo han sido las intervenciones? ¿En qué dirección han ido: al individuo, a las relaciones o al grupo como unidad? ¿Hacia qué elementos se han enfocado las devoluciones y para qué? ¿Qué hipótesis has formulado para un análisis diagnóstico del momento grupal y del proceso? ¿Qué preguntas, dudas y aspectos vas a llevar a la supervisión? ¿Qué previsión de estrategias de acompañamiento vas a tomar? ¿Sobre qué elementos vas a incidir? ¿Qué aspectos vas a trabajar, en cuanto a las relaciones y en cuanto a cualquiera de los elementos de este esquema que requiera atención? (Ejemplo: voy a trabajar sobre las normas que son muy rígidas o sobre un conflicto latente).
17. Notas del/la observador/a o de los/as observadores/as.

\section{Conclusiones: ventanas para la reflexión y puentes}

Quizás todos deberíamos preguntarnos: ¿se hacen grupos en mi institución?, ¿qué grupos hemos probado?, ¿qué necesitan las personas en relación con los grupos? Si les preguntamos ¿qué nos dirían? Si proponemos ¿qué se haría grupos de qué y para qué?, ¿hay grupos que no veo, que me asustan y que por pereza y miedo no planteo?, ¿me permito poner mis capacidades personales al servicio del grupo: la informática, la danza, la música, el deporte, las artes etc.?

¿Qué necesitas, qué te pasa? ¿Les preguntamos a los ciudadanos qué podemos ofrecerles? Les respondemos como profesional-institución ¿demanda, necesidad, deseo, expectativa, motivación de quién? Grupos abiertos, semiabiertos, socioterapéuticos ¿de qué tipo, para qué y por qué hacerlos?, ¿qué excusas me pongo para no proponer grupos? Si lo propusiera, ¿qué grupo sería? ¿ y si me dejo fantasear? Ya sé que no hay tiempo ¿y si lo saco? Una vez decididos me siento en el círculo con el grupo y le pregunto: ¿qué podemos hacer en común? ¿cuáles son nuestros objetivos? Y me pregunto: ¿cómo coordino la tarea del grupo y las relaciones afectivas?, ¿qué metodología planteo, qué técnicas, qué estrategias?, ¿qué mapas teóricos me ayudan a transitar el territorio del grupo?, ¿hacia dónde voy a orientar mi formación?, ¿con quién me superviso?, ¿con qué capacidades cuento?, ¿cuáles mis limitaciones?, ¿cuáles son mis escenas temidas?, ¿qué actitudes facilitadoras y obstaculizadoras?, y ¿qué aspectos más negativos de mi carácter tengo que saber manejar al coordinar el grupo?

Nos encontramos pronto en la plaza creando presencia y vínculo, en las salas de los centros, en los seminarios de la universidad. También, en el parque de El Retiro de Madrid, por qué no. Es territorio comunitario y los sin-hogar me enseñaron a romper con la rigidez de los contextos, al ofrecerme sus propuestas de salir al «aire», al campo, a comer con la parrilla. Eran épocas en las que todavía se podía tocar los tambores en grupo en el parque, pasábamos tardes en El Retiro, recogiendo materiales para 
sus cuadros. ¡Qué saludable! ponernos en círculo bajo los cedros del parque y encontrarnos con José Antonio, Guillermina, Rosalín, Manolo, José Luis, Francisco, Eulogio, Justo, etc., «huéspedes del aire» a quienes les agradezco haberme enseñado a caminar juntos, en un universo basado en la reciprocidad.
Si no sales al bosque, jamás ocurrirá nada. El río siempre pedía que lo visitaran después del anochecer, los campos necesitaban que alguien los recorriera para poder expresarse en susurros y las historias necesitaban que las contaran fuera del alcance del oído (Pinkola).

\section{Referencias bibliográficas}

Anzieu, D. et al. (1971). La dinámica de los grupos pequeños. Buenos Aires: Kapelusz.

Arija, B. y Herrero, I. (1998). Trabajo Social de grupo con personas sin hogar: de la soledad al vínculo. En Cuadernos de Trabajo Social 11, 181-201.

Arija, B, (2001). Reflexiones de la observadora. En T. Zamanillo, Teoría y práctica del aprendizaje por interacción en grupos pequeños. www.ucm.es/BUCM/tesis/cps/ucm-t25724.pdf.

Arija, B. (2004). Trabajo Social con grupos. Un proceso de vinculación a través del arte. En Revista Trabajo Social Hoy, Monográfico Intervención con grupos (pp. 91-113). Madrid: Colegio Oficial de Diplomados en Trabajo Social de Madrid.

Bales, R. (1970). Personality and interpersonal behaviour. Nueva York: Holt Rinehart and Winston,

Benne, K.D. y Sheats, P. (1948). Functional roles of group members. Journals of ;Social Issues, 2, pp. 42-47.

Bion W.R. (1980). Experiencias en grupos. Barcelona: Paidós.

Bourdieu, P., Chaboredon, J.C. y Passeron, J.C. (1976). El oficio del sociólogo. Madrid: Ed. Siglo XXI.

Bowen. M. (1991). De la familia al individuo. Barcelona: Paidós.

Bowlby, J. (1989). Una base segura. Barcelona: Paidós.

Cabrera, P. (1998) «Huéspedes del aire». Sociología de las personas sin hogar en Madrid. Madrid: UPCO.

Corominas, J. (2006). Breve diccionario etimológico de la lengua castellana. Madrid: Gredos.

García, L. (2012). El Grupo operativo como método participativo: poder y aprendizaje en la relación profesional. Cuadernos de Trabajo Social, 25, 205-219.

Howe (1997) La teoría del vínculo afectivo para la práctica del Trabajo Social. Barcelona: Paidós.

Kisnerman, N. y Mustieles, D. (1997). Sistematización de la práctica con grupos. Buenos Aires: Ed. Lumen-Hvmanitas.

Laplanche, J. y Pontalis, J. B. (1994). Diccionario de psicoanálisis. Barcelona: Labor.

Lewin, K. (1988). La teoría de campo en la ciencia social. Barcelona: Paidós.

López- Yarto, L. (1997). Dinámica de grupos: cincuenta años después. Bilbao: DDB.

Marinas, J. M. (2000). La fábula del bazar: orígenes de la cultura del consumo. Madrid: Antonio Machado.

Moreno, J. L. (1966). Psicoterapia de grupo y psicodrama. México: Fondo de Cultura Económica.

Napier, R. W. et al. (2000). Grupos: teoría y experiencia. México: Trillas.

Navarro, S. (2004). Redes sociales y construcción comunitaria. Madrid: CCS.

Peñarrubia, F. (1998). Terapia Gestalt. La vía del vacio fértil. Madrid: Alianza Editorial.

Pichon-Rivière, E. (1985). Del psicoanálisis a la psicología social I. Buenos Aires: Nueva Visión.

Popper, K. (1982). La lógica de la investigación científica. Madrid: Tecnos.

Rossell, T. (1998). Trabajo social de grupo: grupos socioterapéuticos y socioeducativos. Cuadernos de Trabajo Social, 11, 103-122.

Rogers, C. (1961). El Proceso de Convertirse en Persona. Barcelona: Paidós.

Rogers, C. (1973). Grupos de encuentro. Buenos Aires: Ed. Amorrortu.

Spitz, R. (1999). Primer año de la vida del niño. Madrid: Fondo de Cultura Económica de España.

Schutz, W. (2001). Todos somos uno, la cultura de los encuentros. Buenos Aires: Amorrortu.

Valiente, D. (1987). Psicoterapia psicoanalítica de grupos. Madrid: Ed Fundamentos.

Vals, J (1995). Diccionario Freudiano Yébenes. Madrid.

Wazlawick, P. et al. (1981). Teoría de la comunicación humana. Barcelona: Ed. Herder. 
Zamanillo, T. (2008). Trabajo social con grupos y pedagogía ciudadana. Madrid: Síntesis.

Zamanillo, T. (dir.) (2011). Ética, teoría y técnica la responsabilidad política del trabajo social. Madrid: Talasa. 